\title{
Comparison of Activities of Tibialis Anterior, Peroneus Longus, and Tibialis Posterior Muscles according to Lunge Squats and Bulgarian Split Squats in a Healthy Population
}

\author{
Yong-wook Kim, Ph.D.; Tea-heon Kim, BSc; Mi-na Yang, BSc; Ye-seul Yon, BSc; Ji-hye Lee, BSc \\ Department of Physical Therapy, College of Medical Sciences, Jeonju University, Jeonju, South Korea
}

Background Previous studies reported that one-leg squat has various advantages in functional activities, no study has studied the effect of muscle activity on ankle stability.

Purpose The purpose of this study was to investigate the differential effects of squat type on the activity of the tibialis anterior, peroneus longus and tibialis posterior muscle for ankle stability.

Study design Cross-sectional study.

Methods The participants were 30 healthy adults. During each squat execution, tibialis anterior, peroneus longus and tibialis posterior muscle activities were assessed using surface electromyography under control of each squat posture.

Results The muscle activity of the tibialis anterior and peroneus longus applying Bulgarian split squat was significantly higher than lunge squat. However, the activity of the tibialis posterior showed no significance between squat conditions.

Conclusions This study demonstrated that Bulgarian squat was more effective at eliciting ankle dorsiflexor and evertor that are known as important role for ankle stability.

Key words Ankle stability; Bulgarian squat; Electromyography; Peroneus longus; Tibialis anterior.
Journal of KEMA

2017; 1(1): 26-30

Published Online

Dec 30, 2017

pISSN: 2586-4351

Article History

Received 21 November 2017 Revised 27 November 2017

Accepted 27 November 2017

\section{CONTACT}

ptkim@jj.ac.kr

Yong-wook Kim,

Department of Physical

Therapy, College of Me-

dical Sciences, Jeonju Uni-

versity, Jeonju, Republic of Korea

This is an Open-Access article distributed under the terms of the Creative Commons Attribution Non-Commercial License (http://creativecommons. org/licenses/by-nc/4.0) which permits unrestricted noncommercial use, distribution, and reproduction in any medium, provided the origina work is properly cited.

\section{INTRODUCTION}

More than $85 \%$ of ankle sprains that occur frequently in clinical practice are caused by excessive inversion of ankles. ${ }^{1}$ Repeated ankle sprains can lead to chronic ankle instability (CAI). ${ }^{2}$ Causes of CAI are looseness of lateral ligaments of the ankle, deterioration of proprioceptive sensation, decrease of posture control ability, and muscle weakness after ankle sprain. ${ }^{3}$ Dynamic ankle stability is defined as the ability to maintain balance in response to disturbing forces from the outside. ${ }^{4,5}$ Stabilization of dynamic joints is achieved by contraction of muscles sur- rounding joints. Eccentric activity of calf muscles and concentric activity of tibialis anterior and posterior muscles are important for ankle stability. ${ }^{6,7}$ As a method to prevent CAI, strengthening of peroneus longus that, contributes to eversion of the ankle joint has been, suggested. ${ }^{8}$

Among therapeutic interventions to prevent CAI, squat exercises are typical closed-chain movements that occur simultaneously with movements of ankle, knee, and hip joints. They can be properly performed through overall biomechanics and neuromuscular control. ${ }^{9,10}$ Squat exercises are also important to maintain good alignment of ankle posture. They have been recommended for the management 
of CAI. ${ }^{11}$ Previous studies have shown that one leg squat requires more neuromuscular activity due to reduced mediolateral base of support compared to two leg squats. ${ }^{11-13}$ Increased neuromuscular activity has also been reported to have a positive effect on proprioception and strength in functional activities. ${ }^{11-13}$ Although many studies have reported that one-leg squat has various advantages in functional activities, no study has studied the effect of muscle activity on ankle stability.

Therefore, the objective of this study was to investigate the effect of two types of single-leg squat exercise commonly used in clinical practice for strengthening and rehabilitation of lower limbs on muscles activity of the tibialis anterior (TA), peroneus longus (PL), and tibialis posterior (TP). The hypothesis of this study was that muscle activities of TA, PL, and TP muscles at Bulgarian split squat could be higher than those at lunge squat.

\section{METHODS}

This study recruited 30 healthy volunteers (15 males, 15 females) who met the selection criteria. Participants were given a detailed explanation of the study procedure. Written informed consent was obtained from each participant. This study was conducted in accordance with principles of the Declaration of Helsinki.

Volunteers who were able to maintain single-leg squat posture for longer than $10 \mathrm{~s}$ to check shank muscles activity were recruited for this study. Volunteers who had any neurological, musculoskeletal, or cardiopulmonary problems were excluded. True leg length was used to set the distance between the front and back feet during squatting. True leg length was defined as a distance from the anterior posterior iliac spine of the pelvis to the medial malleolus. Mean age, height, weight, and leg length of these participants were $22.9 \pm 1.7$ years, $168.5 \pm 11.1 \mathrm{~cm}, 64.0 \pm 12.9 \mathrm{~kg}$, and $85.1 \pm$
$5.9 \mathrm{~cm}$, respectively.

Before measuring electromyography (EMG) signals and attaching electrodes, any hair on the skin to be attached was shaved. The skin was then cleaned with an alcohol swab before attaching the electrodes. ${ }^{14}$ EMG electrodes were attached to the TA, PL, and TP according to published recommendations. ${ }^{14}$ A Delsys Trigno EMG system (Delsys, Inc, Wellesley, MA, USA) was used to collect EMG data. EMG signals were converted to digital signals and processed using Works Acquisition EMG analysis software for personal computers (Delsys, Inc, Wellesley, MA, USA). The sampling rate of EMG signals was $2,000 \mathrm{~Hz}$ and EMG frequency bandwidth was restricted to $20-500 \mathrm{~Hz}$. The common mode rejection ratio was set at $110 \mathrm{~dB}$. The application of each squat was in random order. Metronome application was used to keep squat posture time constant. EMG measurement and operation of the metronome were started at the same time. After the start of the measurement, a squat posture was made for three seconds at the start position. The squat posture was maintained for five seconds. It was then returned to the start position for three seconds. EMG measurement value analysis and processing were performed using root mean square (RMS) value for five seconds to keep the posture except for the first and last one for three seconds. For Bulgarian split squat, the meta-tarsophalangeal joint of the left foot was placed on the support board which was $30 \%$ length of each subject's height. The hip joint was allowed to extend 10 degrees. During the squat, the squat depth was adjusted so that the femur and tibia of the right leg were at an angle of 90 degrees and the right knee moved forward only to the position of the right toe (Figure 1). The starting position of the lunge squat was the same as the Bulgarian split squat. In order to maintain the trunk in upright posture, each hand was placed on the iliac crest. During lunge squat, the left knee was placed about two inches above the floor while the right leg was perpendicular to the

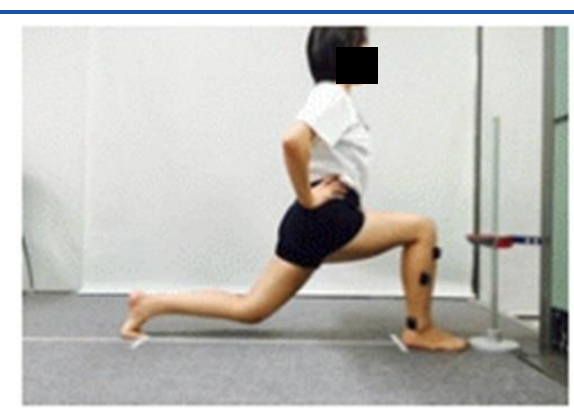

(A)

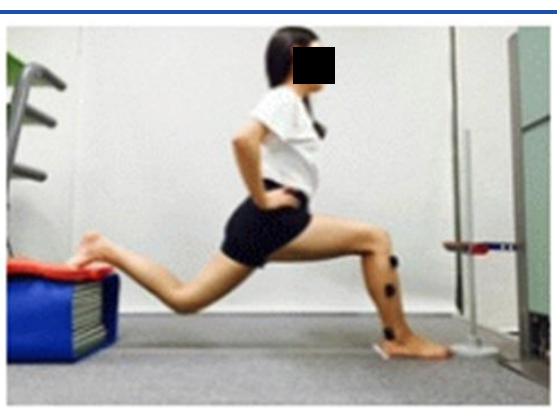

(B)

Figure 1. Experimental posture used to measure tibialis anterior, peroneus longus and tibialis posterior muscle activity during lunge squat (A) and Bulgarian split squat (B). 


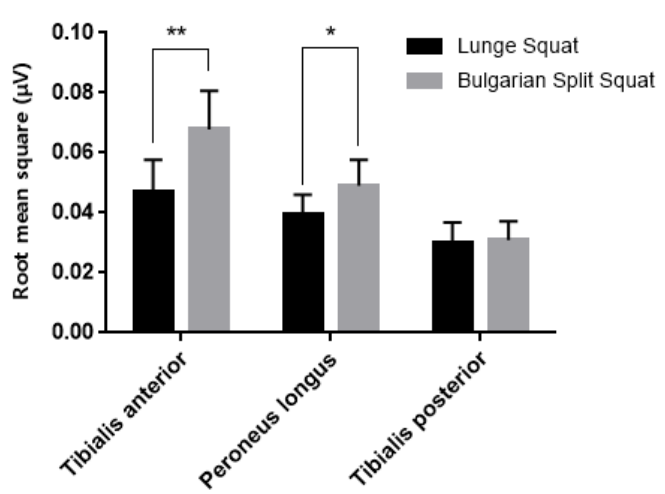

Figure 2. Muscle activity during Bulgarian split squat and lunge squat in the same group $(* p<0.05$, ** $p<0.01)$.

floor.

To prevent muscle fatigue, subjects were allowed a 2-min break between measurements. RMS of EMG was used to measure the activity of each shank muscle. Three measurements were taken for each squat and the mean value of RMS of each muscle was calculated.

All analyses were conducted using SPSS (ver. 21.0; IBM, Armonk, NY, USA). Kolmogorov-Smirnov test was used to confirm that the data were distributed normally. Paired $t$-test was used to compare differences in muscle activity during each squat condition. Statistical significance was accepted at $p<0.05$.

\section{RESULTS}

There were significant differences in the EMG activities of the TA $(t$-value $=7.591, p<0.01)$ and PL $(t$-value $=2.771$, $p<0.05$ ) between squat conditions (Figure 2). During $\mathrm{Bu}-$ lagrian split squat, EMG activities of the TA and PL were significantly higher than those at lunge squat. In contrast, the RMS value of the TP showed no significant difference between the two squat conditions ( $t$-value $=0.303, p>0.05)$ (Figure 2).

\section{DISCUSSION}

Squat activity is achieved through cooperative contraction of the hip, knee, and ankle muscles. Various types of squat exercise have been used in physical training and rehabilitation of individuals with CAI or knee joint deformities such as knee valgus and patellofemoral pain. ${ }^{15-17}$ This study was conducted to examine the effect of two types of squat on muscle activities of the anterior tibialis ankle, long calf muscle, and tibialis ankle contributing to the stability of the ankle joint. As a result of this study, when Bulgarian split squat was applied compared to lunge squirt, muscle activi- ties of TA and PL muscle were increased significantly $(p<0.05)$. This result can be explained by increased tendency to support body weight with anterior leg during the Bulgarian split squat, thereby increasing the contraction force of TA and PL muscles important for ankle stability.

The TA muscle is used as the main actor of ankle dorsiflexion during squat. It occurs as a closed chain movement in the ankle joint. During squat, anterior-posterior ankle stability is mainly dependent on alternating activity of the TA and medial gastrocnemius. ${ }^{18-19}$ When the anterior leg moves forward beyond the vertical line, the eccentric contraction of calf muscle is increased while the activity of TA muscle is increased when moving backward during squat exercise. ${ }^{20}$ The role of PL muscle is to maintain ankle stability by preventing excessive ankle inversion. ${ }^{21}$ Supination of the rear foot that occurs during squatting contributes to dynamic stability of the ankle complex through eccentric contraction of PL and peroneus brevis muscles. In particular, the PL muscle limits ankle inversion and plantarflexion to prevent damage to lateral ligaments of the ankle joint. ${ }^{21}$ The TP muscle is the main inverter of the ankle joint. It is a dynamic stabilizer that supports the medial longitudinal arch. ${ }^{22}$ In the present study, there was no significant difference in average value of TP muscle activity between the two squat conditions. The average value of TP muscle activity during lunge squat was $0.029 \mathrm{mV}$. It was $0.031 \mathrm{mV}$ during Bulgarian split squat. The reason for this is that the muscle activity of TP during squat movement (which is mainly activated by plantarflexion and inversion of the ankle joint) is relatively low during dorsiflexion and eversion of the ankle joint.

Hip extension occurred more during Bulgarian split squat than that during lunge squat. This caused the body to move forward and naturally increased weight bearing of the anterior foot while the center of pressure was located at the forefoot. Therefore, muscle contractions of the ankle dorsiflexor and everters contributing to ankle stability during Bulgarian split squat seemed to be increased more than that during lunge squat.

This study has several limitations. Although we used a predetermined target frame to control the squat exercise, the speed of squat movement which might affect EMG signals was not controlled for each subject. In addition, surface EMG was used to monitor muscle activity, leaving the possibility of crosstalk from adjacent muscles. Therefore, further studies are needed to verify the effect of various squat exercises on the activity of leg muscles in individuals with musculoskeletal abnormalities of the lower extremity. 


\section{CONCLUSIONS}

This study investigated the effect of ankle stabilization on lunge squat and Bulgarian split squat to compare muscle activities of the TA, PL, and TP. Muscle activities of the TA and PL during Bulgarian split squat were significantly higher than those during lunge squat. However, there was no significant difference in muscle activity of the TP between the two squat conditions. Therefore, the hypothesis of this study was partially confirmed in that the Bulgarian split squat showed higher ankle stability than lunge squat.

\section{Key Points}

Question Can Bulgarian squat exercise increase more muscle activity of tibialis anterior, peroneus longus, and tibialis posterior which are contribute the ankle stability?

Findings Muscle activity of the tibialis anterior and peroneus longus applying Bulgarian split squat was higher than applying lunge squat.

Activity of the tibialis posterior showed no difference between squat conditions.

Meaning Bulgarian split squat was more effective to elicit ankle dorsiflexor and evertor for ankle stability.

\section{Article information}

Conflict of Interest Disclosures: None.

Funding/Support: None.

Acknowledgment: None.

\section{REFERENCES}

1. Eils E, Rosenbaum D. A multi-station proprioceptive exercise program in patients with ankle instability. Med Sci Sports Exerc. 2001;33(12):1991-1998.

2. Hertel J. Functional anatomy, pathomechanics, and pathophysiology of lateral ankle instability. $J$ Athl Train. 2002;37(4):364-375.

3. Freeman MA, Dean MR, Hanham IW. The etiology and prevention of functional instability of the foot. $J$ Bone Joint Surg Br. 1965;47(4):678-685.

4. Iwamoto Y, Takahashi M, Shinkoda K. Differences of muscle co-contraction of the ankle joint between young and elderly adults during dynamic postural control at different speeds. J Physiol Anthropol. 2017;36(1):32.

5. Kim MK, Yoo KT. The effects of open and closed kinetic chain exercises on the static and dynamic balance of the ankle joints in young healthy women. J Phys Ther Sci.
2017;29(5):845-850.

6. Thompson C, Schabrun S, Romero R, Bialocerkowski A, Marshall P. Factors contributing to chronic ankle instability: a protocol for a systematic review of systematic reviews. Syst Rev. 2016;5:94.

7. Kobayashi T, Gamada K. Lateral ankle sprain and chronic ankle instability: A critical review. Foot Ankle Spec. 2014;7(4):298-326.

8. Kim Y, Kim E, Song Y, Han D, Richards J. The effects of functional instability of the ankle joint on balance. Physiother Pract Res. 2016; 37(1):3-9.

9. Salem GJ, Salinas R, Harding FV. Bilateral kinematic and kinetic analysis of the squat exercise after anterior cruciate ligament reconstruction. Arch Phys Med Rehabil. 2003;84(8):1211-1216.

10. Giatsis G, Kollias I, Panoutsakopoulos V, Papaiakovou G. Biomechanical differences in elite beach-volleyball players in vertical squat jump on rigid and sand surface. Sports Biomech. 2004;3(1):145-158.

11. Nairn BC, Sutherland CA, Drake JD. Motion and muscle activity are affected by instability location during a squat exercise. J Strength Cond Res. 2017;31(3):677685.

12. Severin AC, Burkett BJ, McKean MR, Wiegand AN, Sayers MGL. Quantifying kinematic differences between land and water during squats, split squats, and single-leg squats in a healthy population. PLoS One. 2017; 12(8):e0182320.

13. Graci V, Van Dillen LR, Salsich GB. Gender differences in trunk, pelvis and lower limb kinematics during a single leg squat. Gait Posture. 2012;36(3):461-466.

14. Surface ElectroMyoGraphy for the Non-Invasive Assessment of Muscles Web site [internet]. Recommendations for sensor locations on individual muscles; [cited 2015 Mar 1]. Available from: http://www.seniam.org

15. Graci V, Salsich GB. Trunk and lower extremity segment kinematics and their relationship to pain following movement instruction during a single-leg squat in females with dynamic knee valgus and patellofemoral pain. J Sci Med Sport. 2015;18(3):343-347.

16. Lewis CL, Foch E, Luko MM, Loverro KL, Khuu A. Differences in lower extremity and trunk kinematics between single leg squat and step down tasks. PLoS One. 2015;10(5):e0126258.

17. Scattone Silva R, Serrão FV. Sex differences in trunk, pelvis, hip and knee kinematics and eccentric hip torque in adolescents. Clin Biomech (Bristol, Avon). 2014;2 (9):1063-1069.

18. Almeida GL, Carvalho RL, Talis VL. Postural strategy to keep balance on the seesaw. Gait Posture. 2006; 
23(1):17-21.

19. Maeda N, Urabe Y, Fujii E, Moriyama N, Iwata S, Sasadai J. The effect of different stretching techniques on ankle joint range of motion and dynamic postural stability after landing. J Sports Med Phys Fitness. 2016;56 (6):692-698.

20. Williams VJ, Nagai T, Sell TC, et al. Prediction of dynamic postural stability during single-leg jump landings by ankle and knee flexibility and strength. J Sport
Rehabil. 2016;25(3):266-272.

21. Ashton-Miller JA, Ottaviani RA, Hutchinson C, Wojtys EM. What best protects the inverted weight bearing ankle against further inversion? Evertor muscle strength compares favorably with shoe height, athletic tape, and three orthoses. Am J Sports Med. 1996;24(6):800-809.

22. Moon D, Park H, Choi E, Kim D, Kim M. Posterior tibialis tendon dysfunction: Preliminary report. $J$ Korean Orthop Assoc. 2008;43(6):799-807. 